\title{
The estrogen receptor signaling pathway activated by phthalates is linked with transforming growth factor- $\beta$ in the progression of LNCaP prostate cancer models
}

\author{
HYE-RIM LEE, KYUNG-A HWANG and KYUNG-CHUL CHOI \\ Laboratory of Biochemistry and Immunology, College of Veterinary Medicine, \\ Chungbuk National University, Cheongju, Chungbuk 361-763, Republic of Korea
}

Received January 14, 2014; Accepted March 27, 2014

DOI: $10.3892 / \mathrm{ijo} .2014 .2460$

\begin{abstract}
The distinct roles of estrogen receptors (ERs) related with androgen receptors (ARs) have been proposed in prostate cancer, while the involvement of transforming growth factor- $\beta$ (TGF- $\beta$ ) has been reported in the progression of prostate cancer. In this study, we examined whether the TGF- $\beta$ signaling pathway is associated with ER signaling in LNCaP prostate cancer cells, which express ER $\alpha, E R \beta$ and ARs. We determined whether the exposure to phthalates may induce prostate cancer progression by affecting molecular crosstalk between ER and TGF- $\beta$ signaling pathways. Cell viability was measured in LNCaP cells by MTT assay following treatment with di-n-buthyl phthalate (DBP). RT-PCR and immunoblot assay were performed to examine the expression levels of cell cycle-related genes and the TGF- $\beta$ signaling cascade. A mouse xenograft model of prostate cancer was generated, and immunohistochemical and BrdU assay were carried out to determine the effect of DBP in this mouse model. DBP, a type of phthalate, was shown to promote $\mathrm{LNCaP}$ cell proliferation by upregulating the gene expression of c-myc and cyclin D1 and by downregulating the expression of p21. DBP significantly reduced the protein expression of p-smad similarly to E2. These regulations caused by DBP were reversed by ICI 182,780, an ER antagonist, indicating that DBP may affect crosstalk between TGF- $\beta$ and ER signals. In an in vivo mouse model, tumor volume of mice exposed to DBP was increased. Number of cells in $S$ phase of cell cycle was increased by DBP, while expression of $\mathrm{p} 21$ protein was reduced in the tissues of DBP-treated mice. These results indicate that DBP may induce the growth of $\mathrm{LNCaP}$ prostate cancer by acting on the crosstalk between TGF- $\beta$ and ER signaling pathways.
\end{abstract}

Correspondence to: Professor Kyung-Chul Choi, Laboratory of Biochemistry and Immunology, College of Veterinary Medicine, Chungbuk National University, Cheongju, Chungbuk 361-763, Republic of Korea

E-mail:kchoi@cbu.ac.kr

Key words: phthalate, prostate cancer, estrogen receptor, transforming growth factor- $\beta, \mathrm{LNCaP}$ cells

\section{Introduction}

The prostate is a major exocrine gland in males, which is involved in sexual developments (1). The prostate organ grows gradually during puberty by stimuli of male hormone androgen in the body (2). Dihydrotestosterone (DHT) is the critical hormone responsible for prostate growth (3). Diagnosis of prostate cancer formation is increasing and has become a common cancer in males (4). Various factors to promote cancer in human have been supposed to be obesity, smoking, and age (5). The mechanism of the key process in prostate cancer has not been clarified yet (6).

Some reports have assumed that the imbalance in hormones such as androgens and estrogens is the main cause of prostate cancer. Estrogens are known to be potential factors in the progression of breast cancer and ovarian cancer expressing estrogen receptors (ERs). These facts suggest that estrogenresponsive organs can be adenocarcinoma by overactivation of ERs signaling and are asserted with evidence of laboratory and clinical research (7). Estrogen-induced signaling directly contributes to modify gene expression to alter normal biological mechanism (8). Especially, the distinct roles of ERs, $\mathrm{ER} \alpha$ and $\mathrm{ER} \beta$, in relation with androgen receptor (AR) have been focused on prostate disease (9). In addition, it has been reported that growth factors or cytokines are involved in the progression of prostate cancer (10) and applied for therapeutic targets to conquer cancer and metastasis into other secondary site (11).

Among growth factors, the growth regulatory proteins of transforming growth factor- $\beta$ (TGF- $\beta$ ) family are endogenous inhibitors of cell growth (12). After binding of three types of TGF- $\beta$ with their receptors, Smad2/3 proteins are phosphorylated and form a complex with Smad4. Smad complex translocates to the nucleus and regulates transcriptional expression of downstream genes by binding DNA as a transcription factor (13). Cancer is characterized by uncontrolled cell proliferation compared to normal cells (14). TGF- $\beta$ is a multifunctional cytokine, which regulates cell proliferation, differentiation and apoptosis of cells in most tissues. For example, null mouse experiments for TGF- $\beta$ have suggested that TGF- $\beta$ plays a role in inflammation. Other studies also showed that TGF- $\beta$ is linked to carcinogenesis (15). 
Variation of mRNA levels of three TGF- $\beta$ correlates with progression of human cancer such as glioma, as determining tumor growth by modification of microenvironment surrounding cancer. TGF- $\beta$ may cause an activation of signaling pathways of other growth factors such as vascular endothelial growth factor (VEGF) and plasminogen activator inhibitor-1 (PAI-1) (16). Carcinogenesis is affected by TGF- $\beta$ as well as the family of Smad. The TGF- $\beta /$ Smad signaling pathway, which is activated in prostate cancer, has a regulatory effect on the cell cycle. TGF- $\beta$ signaling pathway is closely associated with $\mathrm{p} 21$, p27, c-myc and c-fos in cancer (17). c-myc is a cellular protooncogene, with a potential in modification of proliferation and apoptosis of human cancer cells (18). c-myc and c-fos reportedly control TGF- $\beta$ signaling and cell cycles (19). Overexpression of c-myc is associated with cancer initiation and metastasis (20). Family of myc is important in the transformational changes to cancer (21). Myc plays central roles in the activity of cyclin D1-Cdk4 during $\mathrm{G}_{0}$ to $\mathrm{S}$ transition in the cell cycle (22). Abnormality of the cancer cell cycle results in production of cyclin (23) which degrades p21 and p27 (24).

Expressions of the cell cycle-related genes, c-myc, cyclin D and p21, were altered in cancer by endocrine disrupting chemicals (EDCs) via steroid hormone receptor signaling pathways in our previous studies (25-27). EDCs are environmental synthetic chemicals that disrupt the endocrine system $(28,29)$. Accumulation of EDCs in the body may lead to severe reproductive problems in humans (30). As a result of the development of industry, many synthetic chemicals have been identified as EDCs causing the human health problems $(31,32)$. For example, some pesticides are known as EDCs (33). Among EDCs, humans are exposed to phthalate in the environment (34). Phthalates are similar diesters of phthalic acid used as plasticizer to make plastics soft (35). Recently, the production volume of phthalates has increased due to wide applications for plastics (36). Therefore, humans may be easily exposed to phthalate through the plastics, clothes and bottles (25). The impact of phthalate exposure on human health have also been focused on due to toxicity on the reproductive system via androgen and estrogen receptor signaling pathways.

In this study, we determined whether exposure to phthalate may promote prostate cancer in in vitro and in vivo through molecular crosstalk between estrogen receptor (ER) signaling and TGF- $\beta$ signaling pathway. We employed prostate cancer LNCaP cells, which express ER $\alpha, E R \beta$ and ARs. Therefore, these cells are a useful model to study estrogen receptors signaling in prostate cancer.

\section{Materials and methods}

Reagents and chemicals. 17ß-estradiol (E2), di-n-buthyl phthalate (DBP) and ICI 182,780 were purchased from Sigma-Aldrich Corp. (St. Louis, MO, USA). All chemicals were dissolved in $100 \%$ dimethyl sulfoxide (DMSO; Junsei Chemical Co., Tokyo, Japan) and corn oil (Junsei Chemical Co.) 3 days before treatment.

Cell culture and media. LNCaP cells were cultured in Dulbecco's modified Eagle's medium (DMEM; HyClone Laboratories Inc., Logan, UT, USA) supplemented with
$10 \%$ heat-inactivated fetal bovine serum (FBS; HyClone Laboratories Inc.), $1 \%$ penicillin $\mathrm{G}$ and streptomycin (Cellgro; Mediatech, Inc., Manassas, VA, USA), and HEPES (Invitrogen Life Technologies, Carlsbad, CA, USA) at $37^{\circ} \mathrm{C}$ in a humidified atmosphere with $5 \% \mathrm{CO}_{2}-95 \%$ air.

Ablation of estrogenic components. To exclude the effects of estrogenic components in DMEM and FBS, cells were also cultured in phenol red-free DMEM supplemented with 5\% charcoal-dextran stripped FBS (CD-FBS). Cells were detached with $0.05 \%$ trypsin/0.02\% EDTA in $\mathrm{Mg}^{2+} / \mathrm{Ca}^{2+}$-free Hanks' balanced salt solution (PAA Laboratories, Pasching, Austria) before the DBP and E2 treatment.

Cell viability assay. To evaluate the effect of $\mathrm{E} 2$ or DBP on LNCaP cell proliferation, cell viability assay was performed as previously described (32). Cells were seeded at a density of 8,000 cells $/ 100 \mu 1$ of phenol red-free DMEM with $5 \%$ CD-FBS medium per well of 96 well culture plates. After an incubation for $24 \mathrm{~h}$, the cells were washed and treated with various concentrations of chemicals in phenol red-free DMEM supplemented with $0.1 \%$ DMSO for 5 days. DMSO was used as a vehicle and a negative control and E2 as a positive control. Cell viability was detected with the addition of 3-(4-,5-dimethylthiazol-2-yl)-2,5-dyphenyltetrazolium bromide (MTT; Sigma-Aldrich) solution. MTT (10 $\mu \mathrm{l} \mathrm{of} 5 \mathrm{mg} / \mathrm{ml}$ solution) was added to each well and the plates were incubated for $4 \mathrm{~h}$ at $37^{\circ} \mathrm{C}$. Supernatants were removed and $100 \mu 1$ of DMSO was added to each well to dissolve the resultant formazan crystals. The optical density (OD) of each well was measured at $540 \mathrm{~nm}$ using an ELISA reader (Molecular Devices, Sunnyvale, CA, USA) and used to calculate the number of viable cells. All experiments were done at least three times.

Semi-quantitative reverse transcription (RT) PCR. Cells were seeded at a density of $5.0 \times 10^{5}$ cells per well in a 6 -well plate, and then treated with either DMSO, E2 or DBP. Total RNA was extracted using TRIzol reagent (Invitrogen Life Technologies) according to the manufacturer's instructions. cDNA was synthesized from total RNA by reverse transcription (RT). The reaction mixture contained murine leukemia virus reverse transcriptase (M-MLV RT), $200 \mathrm{pM}$ nonamer random primer, dNTPs, RNase inhibitor and RT buffer (all from Intron Biotechnology, Sungnam, Korea). cDNA synthesis was performed at $37^{\circ} \mathrm{C}$ for $1 \mathrm{~h}$ and $95^{\circ} \mathrm{C}$ for 5 min. To analyse the expression of p21, cyclin D1, c-myc and GAPDH, cDNA was amplified by PCR with specific forward and reverse primers, Taq polymerase, PCR buffer and dNTP mixture, and each cDNA template as previously described. The following primers (Bioneer Co., Daejeon, Korea) were used: for cyclin D1, forward (F)-TCTAA GATGA AGGAG ACCAT C and reverse (R)-TGACA GGTCC ACATG GTCTT CC; for p21, F-AGGCA CCGAG GCACT CAGAG and R-TGACA GGTCC ACATG GTCTT CC; and for GAPDH, F-ATGTT CGTCA TGGGT GTGAA CCA and R-TGGCA GGTTT TTCTA GACGG CAG. The PCR products were separated on a $1.5 \%$ agarose gel and the size of each gene band was estimated by comparison with 100-bp size ladders (Intron Biotechnology). The gels were scanned and the band densities were quantified using Gel 
Doc 2000 (Bio-Rad Laboratories, Inc., Hercules, CA, USA). All experiments were done at least three times.

Western blot analysis. To detect protein expression of cyclin D1 and Smad in LNCaP, cells were cultured to a density of $2.0 \times 10^{6}$ cells per of $100-\mathrm{mm}$ dish and then treated with DMSO, E2 or DBP. After treatment, the cells were suspended in $100 \mu \mathrm{l}$ of $1 \mathrm{X}$ RIPA buffer $(50 \mathrm{mM}$ Tris- $\mathrm{HCl}$; pH 8.0, $150 \mathrm{mM} \mathrm{NaCl}, 1 \% \mathrm{NP}-40,0.5 \%$ deoxycholic acid and $0.1 \%$ SDS). Total protein concentrations were determined by bicinchoninic acid (BCA; Sigma-Aldrich Corp.) and $50 \mu \mathrm{g}$ of total protein was then separated by SDS-polyacrylamide gel electrophoresis (SDS-PAGE). The proteins were transferred to a polyvinylidene difluoride (PVDF) membrane (Bio-Rad Laboratories, Inc.), and the membranes were blocked with $5 \%$ bovine serum albumin (BSA; Sigma-Aldrich Corp.) for $2 \mathrm{~h}$ at room temperature. The membranes were incubated with mouse monoclonal anti-Smad (1:500; Santa Cruz Biotechnology, Santa Cruz, CA, USA), rabbit monoclonal anti-pSMAD3 (1:200 dilution; Santa Cruz Biotechnology), mouse monoclonal anti-cyclin D1 or anti-p21 (1:3,000; Cell Signaling Technology, Inc., Danvers, MA, USA), or mouse monoclonal anti-GAPDH (1:1,000; Santa Cruz Biotechnology) antibodies overnight at room temperature. The membranes were subsequently probed with anti-mouse IgG HRP-conjugated secondary antibody (1:1,000; Santa Cruz Biotechnology) for $4 \mathrm{~h}$ at room temperature. Target proteins were detected with a West-Q Chemiluminescent Substrate Plus kit (Gendepot, Barker, TX, USA). All experiments were done at least three times.

Establishment of xenograft prostate cancer models. LNCaP cells $\left(6 \times 10^{6}\right)$ were mixed with Matrigel (BD Biosciences, Bedford, MA, USA) and injected subcutaneously (s.c.) into male nude balb/c mice (5-week-old). Mice were monitored for tumor growth every week, and the tumor volumes were measured using a caliper and expressed by length $\mathrm{x}$ width $\mathrm{x}$ height $\mathrm{x} 0.5236$ $\left(\mathrm{mm}^{3}\right)$. Once tumor reached $50 \mathrm{~mm}^{3}$, the mice were surgically castrated under anesthesia using avertin (Sigma-Aldrich Corp.). The animal experiment was performed according to the protocols approved by the Animal Care Committee of Chungbuk National University. Mice were reinstated for 1 week after surgery, grouped into three groups, and injected s.c. with corn oil (vehicle, $\mathrm{n}=5)$, E2 (n=5; $20 \mu \mathrm{g} / \mathrm{kg} /$ body weight), or DBP ( $\mathrm{n}=5 ; 200 \mathrm{mg} / \mathrm{kg} /$ body weight) every 2 days for 5 weeks (Fig. 1). Cancer tissues were obtained from mice after treatment with chemicals, fixed in $4 \%$ formalin and embedded in paraffin for immunohistochemical analysis.

Immunohistochemistry and bromodeoxyuridine (BrdUrd) incorporation assay. Immunohistochemistry for p21 and bromodeoxyuridine (BrdUrd) was performed for the specimen slides of tumor sections obtained from the mice. Antigen retrieval was achieved in a microwave using $0.01 \mathrm{M}$ citrate buffer, and the slides were treated sequentially with $0.3 \% \mathrm{H}_{2} \mathrm{O}_{2}$, blocking buffer and first antibodies. For detection of expression of target proteins in tissue, biotinylated-mouse anti-goat $\operatorname{IgG}$ (1:1,000 dilution, Vector Laboratories, Inc., Burlingame, CA, USA) was used as a secondary antibody. The primary antibodies used in this assay were a mouse

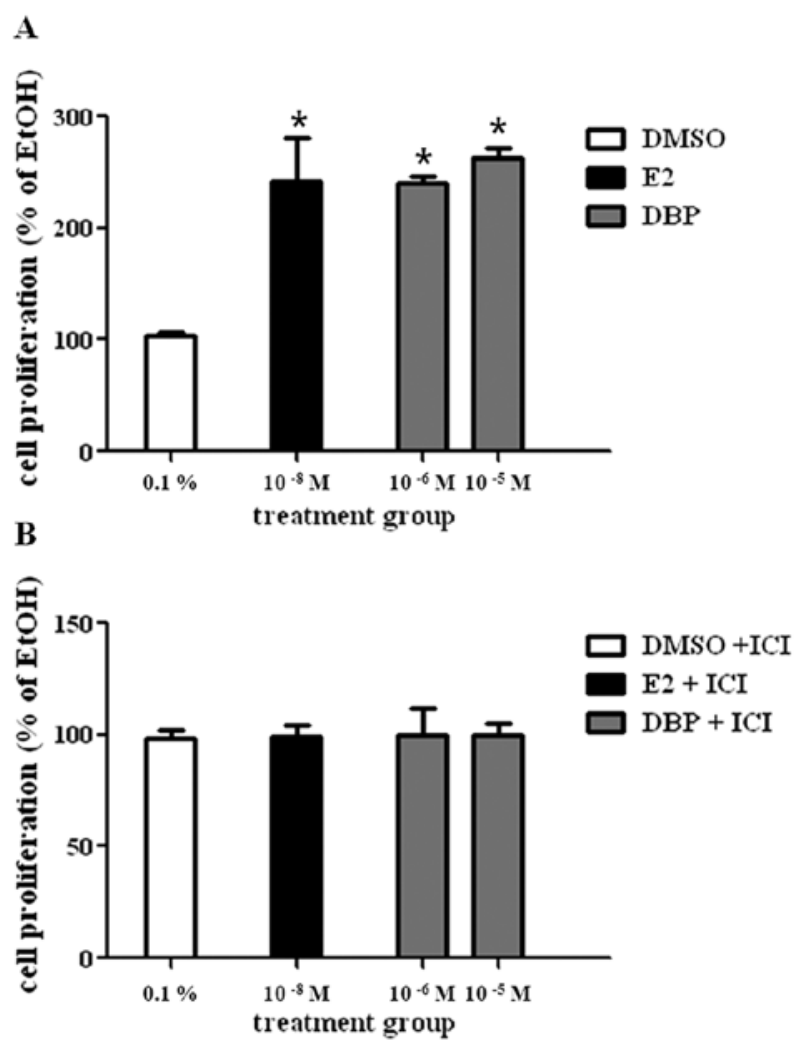

Figure 1. Stimulation effects of phthalate on prostate cancer. To evaluate the effect of E2 or DBP on cell proliferation in LNCaP, cells were cultured with vehicle (0.1\% DMSO, control), E2 $\left(10^{-8} \mathrm{M}\right)$ or DBP $\left(10^{-6} \mathrm{M}\right)$ for 5 days. (A) E2 and DBP statistically increased the growth of prostate cancer cells compared to DMSO. (B) DBP- or E2-induced cell proliferations were reduced by ICI 182,780, ER antagonist. ${ }^{*}$ p $<0.05$ significantly different vs. DMSO.

monoclonal antibody against p21, which is used in the same condition as in western blot analysis, and a mouse monoclonal antibody against BrdUrd (1:100 dilution, Thermo Scientific, Rockford, IL, USA). The tissues were counterstained with hematoxylin (Sigma-Aldrich) and observed using the BX51 microscope (Olympus, Center Valley, PA, USA) for digital photography.

Statistical analysis. All data were analyzed with GraphPad Prism software (San Diego, CA, USA). Data are presented as the mean \pm SD. Statistical analyses were performed using a one-way ANOVA; Dunnett's multiple comparison or Student's t-test. p-values $<0.05$ were considered statistically significant.

\section{Results}

Stimulation effects of phthalate on prostate cancer. To evaluate the effect of E2 or DBP on cell proliferation of LNCaP, cells were cultured with vehicle ( $0.1 \%$ DMSO, control), E2 $\left(10^{-8} \mathrm{M}\right)$, or DBP $\left(10^{-6} \mathrm{M}\right)$ for 5 days. E2 statistically increased the growth of prostate cancer cells compared to DMSO as shown in Fig. 1A. DBP showed a cell proliferation effect similar with E2 at concentrations of $10^{-6}$ and $10^{-5} \mathrm{M}$. DBP-induced cell proliferation was reduced by ICI 182,780 (Fig. 1B), an ER antagonist. This fact may suggest proliferative effect of DBP on LNCaP cells via ER signaling. 

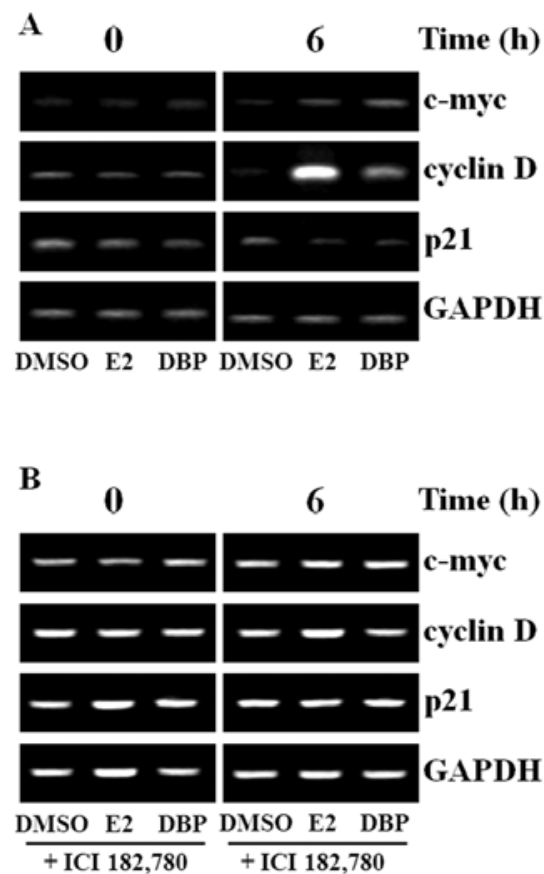
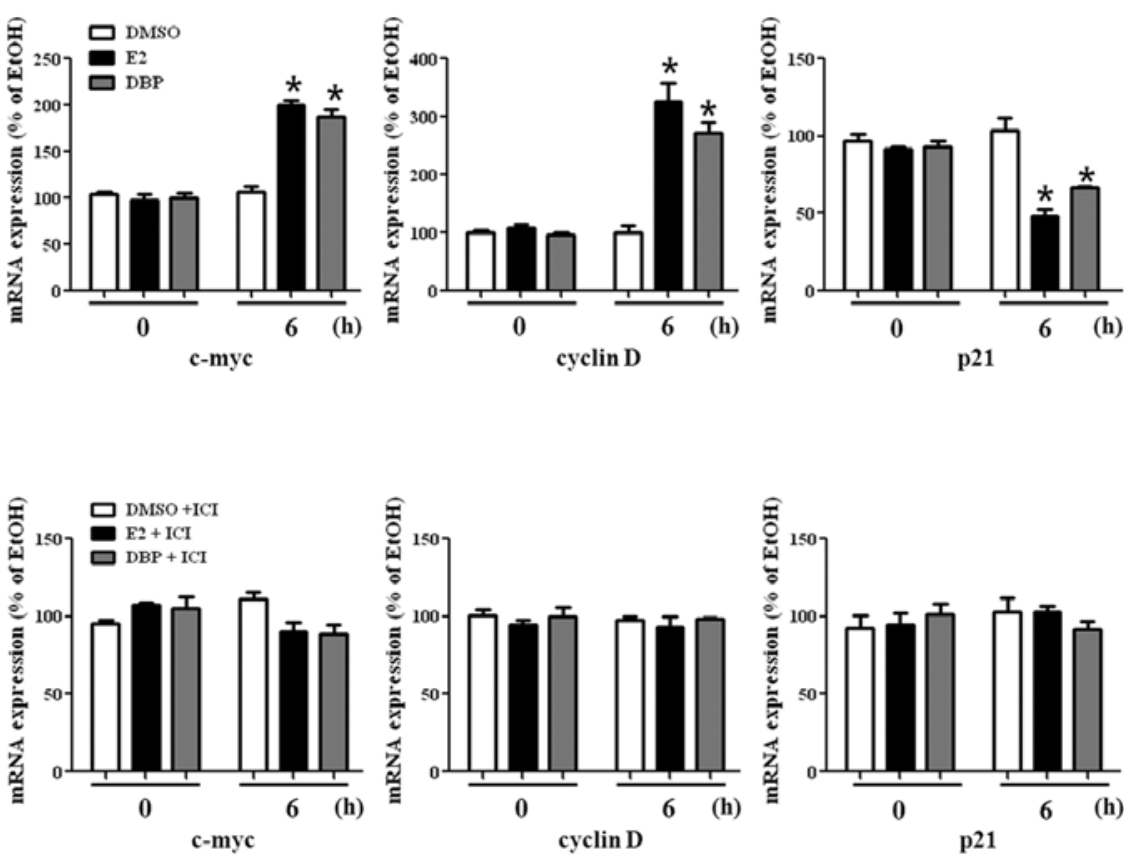

Figure 2. Altered mRNA expression of c-myc, cyclin D1 and p21 in LNCaP cells by exposure to DBP. To perform semi-quantitative RT-PCR on RNA samples, total RNA was isolated from the cells treated with these agents and amplified by PCR. (A) mRNA levels of c-myc and cyclin D1 were significantly increased by treatment with E2 or DBP, while the expression of p21 was markedly decreased compared to the control. (B) Breakdown of ER signaling pathway by the ER antagonist reversed the effects of DBP in LNCaP cancer cells. * $\mathrm{p}<0.05$ significantly different vs. DMSO.

A Time (h)

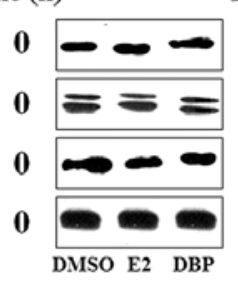

B Time (h)

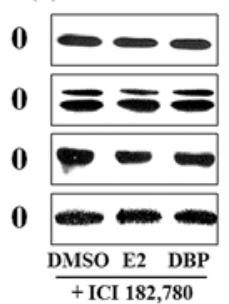

Time (h)

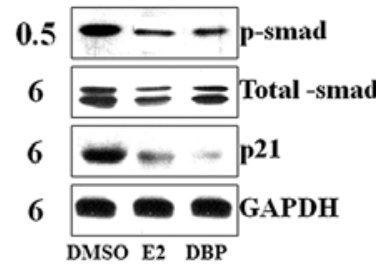

Time (h)

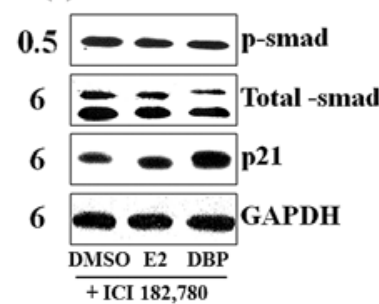

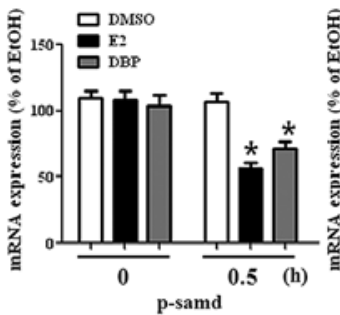
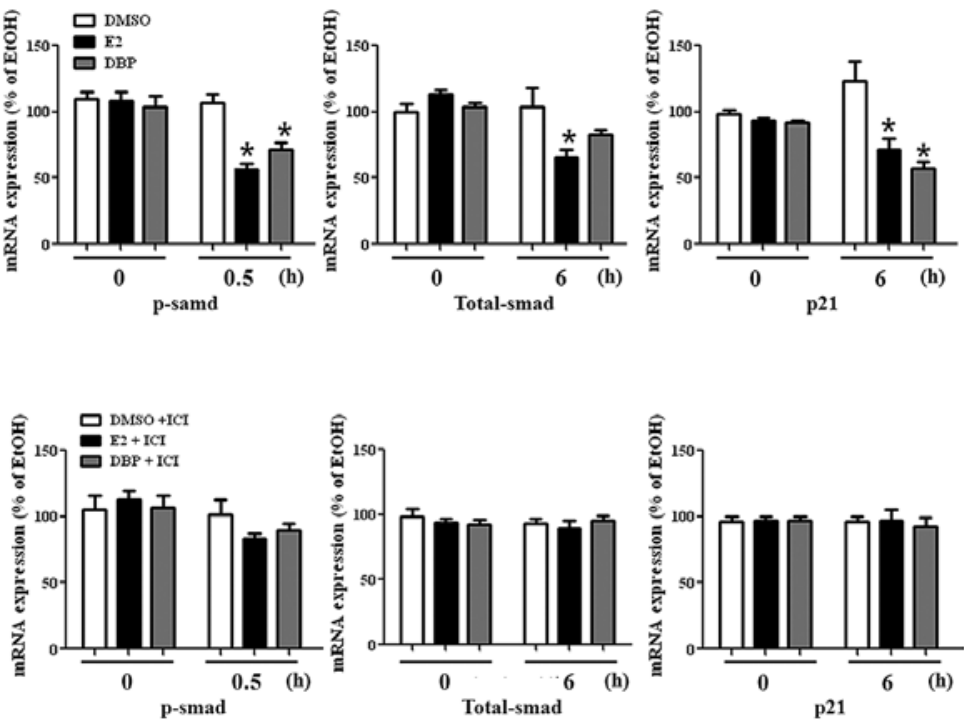

Figure 3. Altered protein expression of smad and p21 in LNCaP cells by exposure to DBP. To detect protein expression of genes, we performed western blot analysis using proteins isolated from LNCaP. (A) The protein expression of p-smad was reduced by $\mathrm{DBP}\left(10^{-6} \mathrm{M}\right)$ in $\mathrm{LNCaP}$ compared to DMSO. In addition, p21 protein was markedly reduced in LNCaP exposed to DBP compared to DMSO. (B) The effects of DBP on protein expression in LNCaP cells were reversed with the addition of ICI 182,780. * p<0.05 significantly different vs. DMSO.

Disruption of $m R N A$ expression regulation of genes by $D B P$. To perform semi-quantitative RT-PCR on total RNA samples, RNA was isolated from the cells treated with these agents and amplified by PCR. First, mRNA levels of c-myc and cyclin D1 were significantly increased by treatment with
E2 or DBP for 6 and $24 \mathrm{~h}$, while expression of $\mathrm{p} 21$ was markedly decreased compared to the control (Fig. 2A). Cyclin D1 and c-myc are promoting effectors, while p21 is an inhibitor in cell cycle progression. Based on MTT assay results, the breakdown of ER signaling pathway by the ER antagonist, 

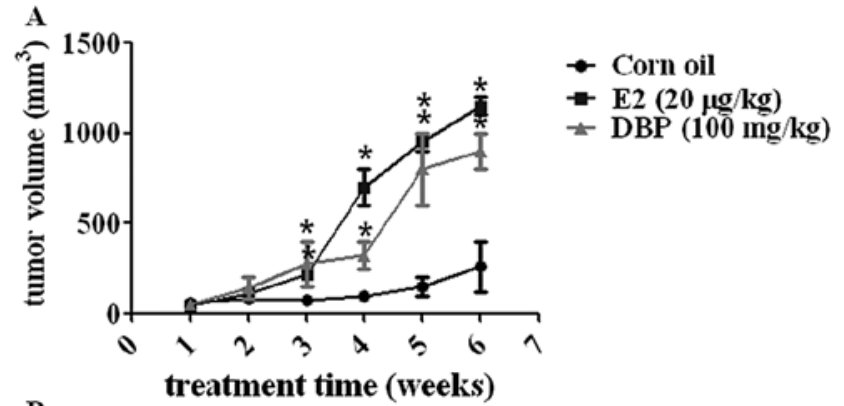

B

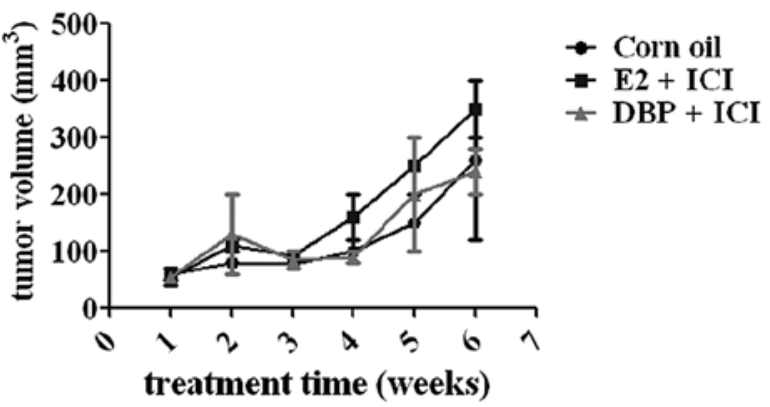

Figure 4. Effect of DBP on tumor growth in vivo. (A) To evaluate the ability of DBP to promote cancer growth, the xenografted male mice transplanted with LNCaP cells were injected s.c. with DBP, E2 or corn oil every other day as indicated in Materials and methods. E2 (a positive control) and DBP markedly stimulated the tumor volume compared to corn oil group (a negative control). (B) Tumor volume did not show statistical results in ICI 182,780 pretreated mice $(\mathrm{p}<0.05) .{ }^{*} \mathrm{p}<0.05$ significantly different vs. treatment with corn oil.

ICI 182,780, reversed the effects of DBP in these cancer cells as seen in Fig. 2B. These results show that phthalates may alter expression of genes related with both TGF- $\beta$ signaling and ER signaling pathway.

Altered protein expression of smad and p21 in LNCaP cells by exposure to $D B P$. To detect protein expression of genes, we performed western blot analysis using protein isolated from LNCaP cells. The expression of $\mathrm{p}$-smad was reduced by DBP as shown in Fig. 3A. The protein expression of p-smad was disrupted following treatment with DBP $\left(10^{-6} \mathrm{M}\right)$. In addition, p21 protein was markedly reduced in LNCaP cells exposed to DBP compared to DMSO, as shown in RT-PCR analysis. However, the suppressive effect of DBP on the expression of these genes in LNCaP was reversed by the addition of ICI 182,780 as demonstrated in Fig. 3B. This fact may imply crosstalk between ER and TGF- $\beta$ signaling pathway because the expression of smads (total smad and p-smad) was influenced by the treatment of E2 and an ER antagonist in prostate cancer cells expressing ER.

Effect of DBP on tumor growth in vivo. To evaluate the ability of DBP to promote cancer growth, the xenografted male mice transplanted with $\mathrm{LNCaP}$ were injected s.c. with DBP, E2 or corn oil every other day as indicated in Materials and methods. E2 (a positive control) and DBP markedly stimulated tumor volume compared to corn oil group (a negative control) as shown in Fig. 4A ( $\mathrm{p}<0.05)$. However, the pre-treatment of the mice with ICI 182,780 reversed the
DBP- or E2-induced increase in tumor volume of prostate cancer xenografted mice (Fig. 4B). This fact demonstrated a critical role of ER-dependent signaling in tumor growth in the mouse models in vivo.

Analysis of cell cycle transition in prostate cancer. The xenografted male mice were injected intraperitoneally (i.p.) with BrdUrd before euthanization and the amount of BrdUrd incorporated into nuclei including the S-phase DNA was detected using immunohistochemistry (Fig. 5). In both E2 and DBP groups, tissues showed significantly increased number of BrdUrd positive nuclei compared with corn oil group (Fig. 5A). These results demonstrated that DBP promotes the growth of prostate cancer as promoting the cell cycle transition. However, pretreatment with ICI 182,780 led to reduced number of BrdUrd positive nuclei in cancer tissue (Fig. 5A). The expression of p21 was reduced in cancer tissue by exposure to DBP similarly to in vitro results (Fig. 5B). DBP-induced expression changes of $\mathrm{p} 21$ were reversed by inhibition of ER signaling pathway (Fig. 5B). These results suggest that the effects of DBP on prostate cancer are dependent on the ER signaling pathway.

\section{Discussion}

Prostate cancer is the most common malignancy diagnosed in men. Androgen signaling is required to affect apoptosis and proliferation of prostate cancer $(37,38)$. Among the factors responsible for formation of cancer, EDCs may adversely impact human health through complex mechanisms, via disruption to endogenous hormone receptor binding (39). In humans, a relation with increased abundance of EDCs and cancer has been reported to focus on the hormone-dependent pathway in cancer formation, especially for estrogen (40).

Phthalates, chemicals for plastic resin, mimic estrogen signaling, resulting in disrupting the action of steroid receptors (26). Humans easily absorb phthalates through the skin or ingest them if food wrapped with plastic film is eaten because phthalates are widely spread in various plastic products as plasticizers. This fact would increase the danger of phthalates that have been reported to have weak estrogenic activity and to compete with endogenous estrogen (26).

An estrogen signaling pathway activates transcription of target genes such as c-myc, cyclin D1 and p21 (41). They are regulated to address the $G_{0} / G_{1}$ and $G_{1} / S$ transition in cells in response to stimuli (42). Furthermore, c-myc may affect other growth factor signaling such as TGF- $\beta$ and ras signaling (43). Both c-myc and cyclin D1 are required for activation of cell growth (44). In addition, c-myc may lead to over-expression of cyclin D1 and down-regulation of p21 (45). In this study, we focused on crosstalk between TGF- $\beta$ and estrogen signaling by DBP in prostate cancer. That LNCaP prostate cancer cells express both ER and AR suggests the critical role of not only AR but also ER signaling pathway during cancer progression (46). Research has shown clinical evidence that estrogen may play an important role in human prostate cancer, as well as in rodent (47). However, the mechanism of estrogen signaling pathway has not been clarified due to its complexity (48).

We performed MTT assays, RT-PCR and western blot analysis using the cellular models in LNCaP cells. Many research groups have used LNCaP cells exposed to E2 (49) 

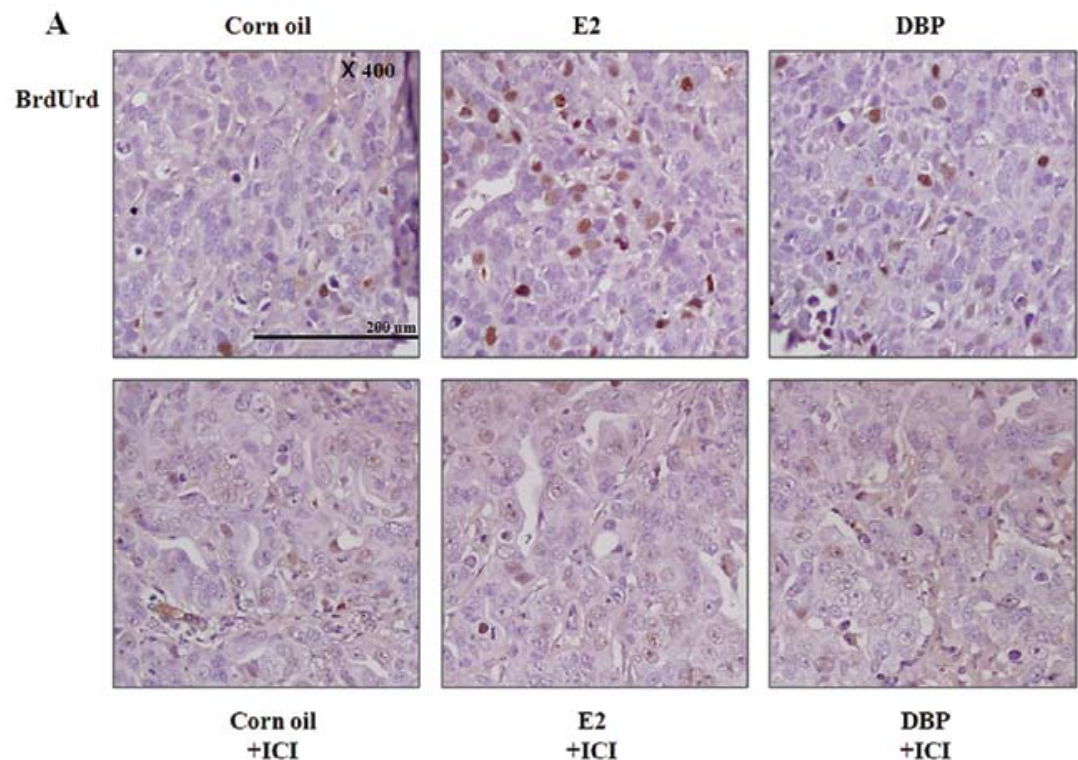

B
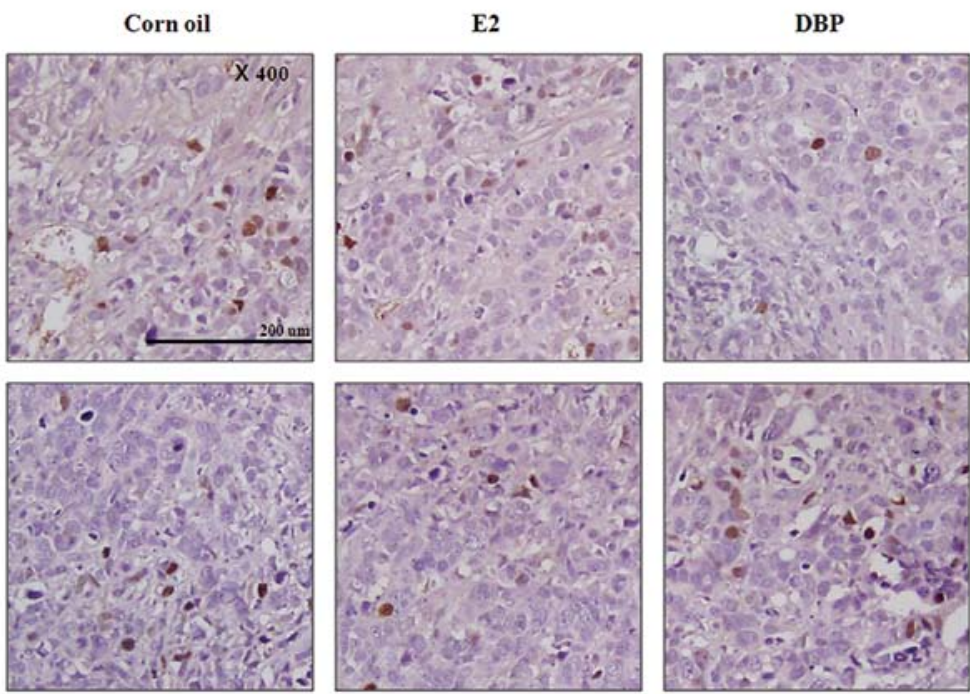

Corn oil

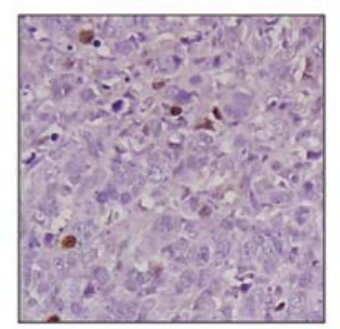

E2

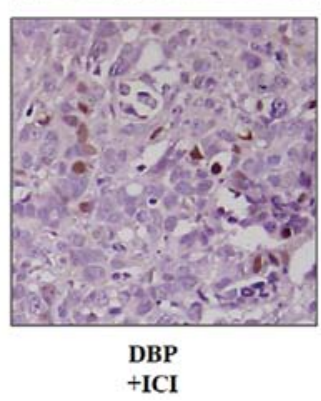

Figure 5. Analysis of cell cycle transition in prostate cancer by DBP. (A) In both E2 and DBP groups, the tumor tissues showed significantly increased number of BrdUrd positive nuclei compared with corn oil group. (B) Pretreatment of mice with ICI 182,780 led to reduced BrdUrd positive nuclei in cancer tissues. The expression of p21 was reduced in cancer tissues by exposure to DBP. DBP-induced changes in p 21 expression were reversed by inhibition of the ER signaling pathway.

showing that estrogen affects epigenesis and cancer (50), and suggesting that ER signaling can be a target for therapy (51). In this study, DBP was shown to promote cell proliferation in $\mathrm{LNCaP}$, while it does not have this effect under the inhibition of ER signaling by ICI 182,780. In addition, c-myc and cyclin D1 expressions were increased and p21 expression was decreased, resulting in cell proliferation. This suggests that DBP may induce cell proliferation by upregulating the gene expression of c-myc and cyclin D1 and by downregulating p21 expression in prostate cancer. The concentrations of DBP in this study were higher compared to E2 because the binding affinity of DBP to ERs appears to be 1,000 times lower compared to that of E2.

An interaction between ER and TGF- $\beta$ signaling pathway was found by the effect of E2 and ER antagonist on the expres- sion of smad, which is an intracellular protein that transduces extracellular signals from TGF- $\beta$ ligands to the nucleus. In western blot analysis, the expression of p-smad was reduced by E2 as well as DBP, while this effect was reversed by the treatment of ICI 182,780 , implying that TGF- $\beta$ signaling is affected by ER signaling, and the DBP mimics E2 action in this interaction. Although the mechanism is not clearly investigated, the result of this study implies that molecular crosstalk between TGF- $\beta$ and ER signaling pathway may have an effect on the stimulation of prostate cancer progression and provides a pathway on which phthalate can act.

The effect of E2 and DBP on prostate cancer growth was confirmed in experimental animals. Tumor volume of mice exposed to E2 and DBP was increased compared to a negative control. These results coincide with the immunohistochemical 
observations in which the number of cells in $\mathrm{S}$ phase was increased by E2 and DBP, while the expression of p21 was reduced in the tissues of E2 and DBP-treated mice. However, the effects of E2 and DBP disappeared in breakdown of ER signaling pathway following the treatment with ICI 182,780, a typical ER antagonist. The amount of injected DBP greatly exceeded E2 in the present study, because EDCs may have an accumulative effect in the body due to continuous exposure and ingestion from an environment unlike endogeneous E2.

In conclusion, DBP, a type of phthalate, may have the potential to promote LNCaP prostate cancer proliferation similarly to E2. Moreover, our results demonstrated that a phthalate acts on crosstalk between TGF- $\beta$ and ER signaling pathway in inducing the growth of prostate cancer. Therefore, this crosstalk can be a target for therapeutic treatment of prostate cancer that can be induced by endogenous hormones or EDCs, including phthalates.

\section{Acknowledgements}

This study was supported by the Priority Research Centers Program through the National Research Foundation of Korea (NRF) funded by the Ministry of Education, Science and Technology (MEST) of Korea government (2009-0094035). In addition, this study was also supported by a grant from the Next-Generation BioGreen 21 Program (no. PJ009599), Rural Development Administration, Republic of Korea.

\section{References}

1. Parnes HL, House MG and Tangrea JA: Prostate cancer prevention: strategies for agent development. Curr Opin Oncol 25: 242-251, 2013.

2. Powers GL and Marker PC: Recent advances in prostate development and links to prostatic diseases. Wiley Interdiscip Rev Syst Biol Med 5: 243-256, 2013.

3. Arai S, Shibata Y, Nakamura Y, et al: Development of prostate cancer in a patient with primary hypogonadism: intratumoural steroidogenesis in prostate cancer tissues. Andrology 1: 169-174, 2013.

4. Dietrich D, Hasinger O, Banez LL, et al: Development and clinical validation of a real-time PCR assay for PITX2 DNA methylation to predict prostate-specific antigen recurrence in prostate cancer patients following radical prostatectomy. J Mol Diagn 15: 270-279, 2013.

5. Meeks JJ and Schaeffer EM: Genetic regulation of prostate development. J Androl 32: 210-217, 2010.

6. Lin D, Bayani J, Wang Y, et al: Development of metastatic and non-metastatic tumor lines from a patient's prostate cancer specimen-identification of a small subpopulation with metastatic potential in the primary tumor. Prostate 70: 1636-1644, 2010.

7. Onita T, Igawa T, Hisamatsu H, Sakai $\mathrm{H}$ and Kanetake $\mathrm{H}$ : Secondary endocrine therapy with oral estrogen for relapsed prostate cancer. Hinyokika Kiyo 55: 595-598, 2009 (In Japanese).

8. Sissung TM, Danesi R, Kirkland CT, et al: Estrogen receptor alpha and aromatase polymorphisms affect risk, prognosis, and therapeutic outcome in men with castration-resistant prostate cancer treated with docetaxel-based therapy. J Clin Endocrinol Metab 96: E368-E372, 2011.

9. Fromont G, Yacoub M, Valeri A, et al: Differential expression of genes related to androgen and estrogen metabolism in hereditary versus sporadic prostate cancer. Cancer Epidemiol Biomarkers Prev 17: 1505-1509, 2008.

10. Holt SK, Kwon EM, Fu R, et al: Association of variants in estrogen-related pathway genes with prostate cancer risk. Prostate 73: 1-10, 2012.

11. Vitkus S, Yeh CR, Lin HH, et al: Distinct function of estrogen receptor alpha in smooth muscle and fibroblast cells in prostate development. Mol Endocrinol 27: 38-49, 2013.
12. Kaminska B, Wesolowska A and Danilkiewicz M: TGF beta signalling and its role in tumour pathogenesis. Acta Biochim Pol 52: 329-337, 2005.

13. Li X, Placencio V, Iturregui JM, et al: Prostate tumor progression is mediated by a paracrine TGF-beta/Wnt3a signaling axis. Oncogene 27: 7118-7130, 2008.

14. Assinder SJ, Dong Q, Kovacevic Z and Richardson DR: The TGF-beta, PI3K/Akt and PTEN pathways: established and proposed biochemical integration in prostate cancer. Biochem $\mathrm{J}$ 417: 411-421, 2009.

15. Jones E, Pu H and Kyprianou N: Targeting TGF-beta in prostate cancer: therapeutic possibilities during tumor progression. Expert Opin Ther Targets 13: 227-234, 2009.

16. Lenferink AE, Cantin C, Nantel A, et al: Transcriptome profiling of a TGF-beta-induced epithelial-to-mesenchymal transition reveals extracellular clusterin as a target for therapeutic antibodies. Oncogene 29: 831-844, 2009.

17. Danielpour D: Functions and regulation of transforming growth factor-beta (TGF-beta) in the prostate. Eur J Cancer 41: 846-857, 2005

18. Miller DM, Thomas SD, Islam A, Muench D and Sedoris K: c-Myc and cancer metabolism. Clin Cancer Res 18: 5546-5553, 2012.

19. Bockelman C, Koskensalo S, Hagstrom J, Lundin M, Ristimaki A and Haglund C: CIP2A overexpression is associated with c-Myc expression in colorectal cancer. Cancer Biol Ther 13: 289-295, 2012.

20. Bouchalova K, Cizkova M, Cwiertka K, Trojanec R and Hajduch M: Triple negative breast cancer - current status and prospective targeted treatment based on HER1 (EGFR), TOP2A and C-MYC gene assessment. Biomed Pap Med Fac Univ Palacky Olomouc Czech Repub 153: 13-17, 2009.

21. Long X, Hu S, Cao P, Liu Z, Zhen H and Cui Y: The expression of oncogene c-myc and its role on human laryngeal cancer. Lin Chung Er Bi Yan Hou Tou Jing Wai Ke Za Zhi 23: 1127-1129, 2009 (In Chinese).

22. Liu M, Casimiro MC, Wang C, et al: p21CIP1 attenuates Rasand c-Myc-dependent breast tumor epithelial mesenchymal transition and cancer stem cell-like gene expression in vivo. Proc Natl Acad Sci USA 106: 19035-19039, 2009.

23. Du YP, Peng JS, Sun A, Tang ZH, Ling WH and Zhu HL: Assessment of the effect of betaine on p16 and c-myc DNA methylation and mRNA expression in a chemical induced rat liver cancer model. BMC Cancer 9: 261, 2009.

24. Hwang KA, Kang NH, Yi BR, Lee HR, Park MA and Choi KC: Genistein, a soy phytoestrogen, prevents the growth of BG-1 ovarian cancer cells induced by $17 \beta$-estradiol or bisphenol A via the inhibition of cell cycle progression. Int J Oncol 42: 733-740, 2012

25. Lee HR, Hwang KA, Park MA, Yi BR, Jeung EB and Choi KC: Treatment with bisphenol A and methoxychlor results in the growth of human breast cancer cells and alteration of the expression of cell cycle-related genes, cyclin D1 and p21, via an estrogen receptor-dependent signaling pathway. Int J Mol Med 29: 883-890, 2012.

26. Park MA, Hwang KA, Lee HR, Yi BR, Jeung EB and Choi KC: Cell growth of BG-1 ovarian cancer cells is promoted by di-n-butyl phthalate and hexabromocyclododecane via upregulation of the cyclin D and cyclin-dependent kinase- 4 genes. Mol Med Rep 5: 761-766, 2012.

27. Park MA, Hwang KA, Lee HR, Yi BR, Jeung EB and Choi KC: Benzophenone-1 stimulated the growth of BG-1 ovarian cancer cells by cell cycle regulation via an estrogen receptor alpha-mediated signaling pathway in cellular and xenograft mouse models. Toxicology 305: 41-48, 2013.

28. Geier R, Adler S, Rashid G and Klein A: The synthetic estrogen diethylstilbestrol (DES) inhibits the telomerase activity and gene expression of prostate cancer cells. Prostate 70: 1307-1312, 2010.

29. Lee HR, Jeung EB, Cho MH, Kim TH, Leung PC and Choi KC: Molecular mechanism(s) of endocrine-disrupting chemicals and their potent oestrogenicity in diverse cells and tissues that express oestrogen receptors. J Cell Mol Med 17: 1-11, 2013.

30. Hess-Wilson JK: Bisphenol A may reduce the efficacy of androgen deprivation therapy in prostate cancer. Cancer Causes Control 20: 1029-1037, 2009.

31. Kang NH, Hwang KA, Kim TH, Hyun SH, Jeung EB and Choi KC: Induced growth of BG-1 ovarian cancer cells by $17 \beta$-estradiol or various endocrine disrupting chemicals was reversed by resveratrol via downregulation of cell cycle progression. Mol Med Rep 6: 151-156, 2012. 
32. Lee HR and Choi KC: 4-tert-Octylphenol stimulates the expression of cathepsins in human breast cancer cells and xenografted breast tumors of a mouse model via an estrogen receptor-mediated signaling pathway. Toxicology 304: 13-20, 2013.

33. Derouiche S, Warnier M, Mariot P, et al: Bisphenol A stimulates human prostate cancer cell migration remodelling of calcium signalling. Springerplus 2: 54, 2013.

34. Mnif W, Hassine AI, Bouaziz A, Bartegi A, Thomas O and Roig B: Effect of endocrine disruptor pesticides: a review. Int J Environ Res Public Health 8: 2265-2303, 2011.

35. Prins GS, Tang WY, Belmonte J and Ho SM: Developmental exposure to bisphenol A increases prostate cancer susceptibility in adult rats: epigenetic mode of action is implicated. Fertil Steril 89: e41, 2008

36. Wetherill YB, Fisher NL, Staubach A, Danielsen M, de Vere White RW and Knudsen KE: Xenoestrogen action in prostate cancer: pleiotropic effects dependent on androgen receptor status. Cancer Res 65: 54-65, 2005.

37. Dulinska-Litewka J, McCubrey JA and Laidler P: Increased Akt signaling resulting from the loss of androgen responsiveness in prostate cancer. Curr Med Chem 20: 144-157, 2013.

38. Lee SO, Ma Z, Yeh CR, et al: New therapy targeting differential androgen receptor signaling in prostate cancer stem/progenitor vs. non-stem/progenitor cells. J Mol Cell Biol 5: 14-26, 2012.

39. Hess-Wilson JK, Webb SL, Daly HK, et al: Unique bisphenol A transcriptome in prostate cancer: novel effects on ERbeta expression that correspond to androgen receptor mutation status. Environ Health Perspect 115: 1646-1653, 2007.

40. Kim SM, Jung EM, An BS, et al: Additional effects of bisphenol $\mathrm{A}$ and paraben on the induction of calbindin-D(9K) and progesterone receptor via an estrogen receptor pathway in rat pituitary GH3 cells. J Physiol Pharmacol 63: 445-455, 2012.

41. Pries R, Hogrefe L, Xie L, et al: Induction of c-Myc-dependent cell proliferation through toll-like receptor 3 in head and neck cancer. Int J Mol Med 21: 209-215, 2008.
42. Serra JM, Gutierrez A, Alemany R, et al: Inhibition of c-Myc down-regulation by sustained extracellular signal-regulated kinase activation prevents the antimetabolite methotrexate- and gemcitabine-induced differentiation in non-small-cell lung cancer cells. Mol Pharmacol 73: 1679-1687, 2008.

43. Guo J, Xiao B, Liu Q, Gong Z and Le Y: Suppression of C-myc expression associates with anti-proliferation of aloe-emodin on gastric cancer cells. Cancer Invest 26: 369-374, 2008.

44. Wang H, Mannava S, Grachtchouk V, et al: c-Myc depletion inhibits proliferation of human tumor cells at various stages of the cell cycle. Oncogene 27: 1905-1915, 2008.

45. Morrish F, Neretti N, Sedivy JM and Hockenbery DM: The oncogene c-Myc coordinates regulation of metabolic networks to enable rapid cell cycle entry. Cell Cycle 7: 1054-1066, 2008.

46. Nakamura Y, Felizola SJ, Kurotaki Y, et al: Cyclin D1 (CCND1) expression is involved in estrogen receptor beta (ER $\beta)$ in human prostate cancer. Prostate 73: 590-595, 2012.

47. Gross M, Ramirez C, Luthringer D, et al: Expression of androgen and estrogen related proteins in normal weight and obese prostate cancer patients. Prostate 69: 520-527, 2009.

48. Nicolaiew N, Cancel-Tassin G, Azzouzi AR, et al: Association between estrogen and androgen receptor genes and prostate cancer risk. Eur J Endocrinol 160: 101-106, 2009.

49. Chae YK, Huang HY, Strickland P, Hoffman SC and Helzlsouer K: Genetic polymorphisms of estrogen receptors alpha and beta and the risk of developing prostate cancer. PLoS One 4: e6523, 2009.

50. Szendroi A, Speer G, Tabak A, et al: The role of vitamin D, estrogen, calcium sensing receptor genotypes and serum calcium in the pathogenesis of prostate cancer. Can J Urol 18: 5710-5716, 2011.

51. Celhay O, Yacoub M, Irani J, Dore B, Cussenot O and Fromont G: Expression of estrogen related proteins in hormone refractory prostate cancer: association with tumor progression. J Urol 184: 2172-2178, 2010 